\title{
Special issue on PDE models and computation: part II
}

\author{
S. Sundar ${ }^{1}$
}

Published online: 5 October 2015

(C) Indian Institute of Technology Madras 2015

Here are another eight interesting original contributions from some of the top-notched researchers in the area of PDE Models and Computation. This issue is largely focussed on the computational aspects and the relevant analysis, in particular to, No Grid Methods (FPM), Finite Element Methods (FEM), Finite Difference Methods (FDM) and Finite Volume Methods (FVM). This set provides again a fine collection of problems and the challenges ahead which in turn throw more challenging research problems.

\section{(1) To Highlight}

Predicting the freezing behavior of SCR dosing system is a direct industrial problem and Dr. Joerg Kuhnert and his team at Fraunhofer ITWM, Kaiserslautern, Germany have brought out the effectiveness of their own developed grid free numerical method, called Finite Pointset Method (FPM), on this important problem.

Canonical von Karman equation with conforming and non-confirming FEM analysis is a new direction of thinking from Prof. Neela Nataraj of IIT Bombay.

Computation over quadtree finite element meshes for strain smoothing is indeed a hard task and that is interestingly brought out by Prof. Sundararajan Natarajan of IIT Madras.
IIT Kanpur Professor Dr. B. V. Rathishkumar, who is considered as one of the leading researcher in the area of parallel computation for flow problems, has presented here an interesting (parallel) computational analysis on the problem of mixed convection with multiple heat source on Anucluster.

Optical flow estimation study is popular among CFD community in recent times. Dr. Praveen Kumar, a CFD specialist from TIFR-CAM Bangalore and his team, have introduced a new variational approach to optical flow estimation for 2D incompressible flows.

Moving from real flow to option pricing, there are two good notable contributions: Improvement of the standard spectral methods for standard option pricing by Prof. Kailash Patidar of University of Western Cape, South Africa and an efficient numerical method for option pricing under jump diffusion model by Prof. Mohan Kadalbajoo of IIT Kanpur.

A novel "entropy stable" central solver for Euler equations has been introduced by Prof. Raghurama Rao of IISc Bangalore.

A few more original contributions are in the lineup. Keep yourself connected to AEAM and enjoy reading good research!
S. Sundar

slnt@iitm.ac.in

1 Department of Mathematics, Indian Institute of Technology, Chennai 600 036, India 